\title{
High serum VE-cadherin and vinculin concentrations are markers of the disruption of vascular integrity during acute aortic dissection
}

\author{
Shiyue Wang \\ First Hospital of China Medical University \\ Yuchen He \\ First Hospital of China Medical University \\ Xin Li \\ First Hospital of China Medical University \\ Shijie Xin \\ First Hospital of China Medical University \\ Jian Zhang ( $\sim$ jianzhang@cmu.edu.cn) \\ First Hospital of China Medical University \\ Han Jiang \\ First Hospital of China Medical University
}

\section{Research Article}

Keywords: vascular-endothelial cadherin, vinculin, acute aortic dissection, endothelial cell, biomarker

Posted Date: January 4th, 2021

DOI: https://doi.org/10.21203/rs.3.rs-135334/v1

License: @ (1) This work is licensed under a Creative Commons Attribution 4.0 International License. Read Full License 


\section{Abstract \\ Background}

In the present study, we measured the serum vascular endothelial cadherin (VEC) and vinculin (Vcn) concentrations in patients with acute aortic dissection (AAD) to evaluate their diagnostic value for this condition.

\section{Methods}

One hundred patients with AAD and 60 matched controls were included in the study. The serum concentrations of VEC and Vcn were measured using enzyme-linked immunosorbent assays.

\section{Results}

The serum VEC and Vcn concentrations were significantly higher in participants with AAD than in healthy controls. Receiver operating characteristic analysis generated areas under the curves for VEC and Vcn that were diagnostic for AAD ( 0.604 and 0.665 , respectively). The optimal cut-off values were $3.986 \mathrm{ng} / \mu \mathrm{L}$ and $128.1 \mathrm{pg} / \mathrm{mL}$, the sensitivities were $43.0 \%$ and $35.0 \%$, and the specificities were $73.3 \%$ and $90.0 \%$, respectively. In addition, the use of a combination of serum VEC and Vcn increased the AUC to 0.739 , with a sensitivity of $56.0 \%$ and a specificity of $76.7 \%$. A high serum Vcn concentration was associated with a higher risk of poor visceral malperfusion in participants with $A A D$ (odds ratio $[O R]=1.007,95 \%$ confidence interval [CI]: $1.001-1.013, p=0.014$ ). In participants with refractory pain, the adjusted OR for the serum VEC concentration increased to $1.172(95 \% \mathrm{Cl}: 1.010-1.361 ; p=0.036)$, compared with participants without refractory pain.

\section{Conclusion}

This study is the first to show the diagnostic value of serum VEC and Vcn for AAD and their relationships with the clinical characteristics of patients with AAD. Thus, VEC and Vcn are potential serum markers of AD.

\section{Introduction}

Acute aortic dissection (AAD) is defined as a disruption of the medial layer of the aorta, which is provoked by intramural bleeding, followed by the separation of the aortic wall layers and the subsequent formation of a true lumen and a false lumen (FL), with or without communication.(1) In most cases, the aortic dissection is initially caused by a tear in the intima, which is followed by the passage of blood into the vascular mesosphere. AADs are commonly divided into type A aortic dissection (TAAD) and type B aortic dissection (TBAD), according to the Stanford classification.(2)

As we all know, computed tomography angiography (CTA) is the gold standard for diagnosing aortic dissection. However, in community hospitals or clinics in China where there is no advanced imaging method, biochemical testing may be the key to help patients make a preliminary diagnosis decision whether to send them home or refer to the tertiary center. Any center needs certain basis for CT examination, and serological screening is its important basis. Compared with CTA, the popularization of serological screening is easier. At the same time, serological examination will be safer and reduce iatrogenic damage for patients who cannot cooperate with computed tomography angiography (CTA) examination or have clear CTA contraindications. Several biomarkers have been shown to be clinically useful for the discrimination of AAD from conditions with similar presentations, such as C-reactive protein (CRP), matrix metalloproteinases (MMPs), soluble elastin fragments (sELAF), D-dimer,smooth muscle myosin heavy chain, calponin, N-terminal pro-brain natriuretic peptide (NTproBNP), big endothelin-1 (Big ET-1), genetic markers and so on.(3)

An increasing number of studies have indicated that the progression of AAD involves a complex pathogenesis, including inflammation, injury to vascular endothelial and smooth muscle cells, and extracellular matrix degradation. It has been widely reported that endothelial integrity plays a crucial role in the maintenance of normal fluid homeostasis and the capacity of the vessel wall to resist thrombosis and inflammatory reactions.(4) Vascular endothelial cadherin (VEC) is a canonical endothelial-specific cell-cell adhesion protein that is critical for endothelial barrier function and the prevention of vascular inflammation. Multiple regulatory and signaling mechanisms converge on VEC and thereby regulate endothelial barrier function and angiogenic remodeling.(5) Vinculin (Vcn) is a ubiquitously expressed cytoskeletal protein that links transmembrane receptors to actin filaments, and plays a key role in the regulation of cell adhesion, motility, and force transmission.(6) Previous studies have shown that the loss of Vcn contributes to the severe weakening of the extracellular matrix and cellcell adhesion, which ultimately promotes cancer proliferation and migration. $(7,8)$ Both VEC and Vcn participate in the establishment of the 
actin cytoskeletal complex, which plays roles in both vascular inflammation and remodeling.(9-11) However, to our knowledge, there is a lack of evidence concerning the relationship between AAD and these two endothelial proteins.

In the present study, we measured the serum VEC and Vcn concentrations in patients with AAD and matched controls and analyzed the clinical relationships between AAD and these two proteins. We also compared the diagnostic value of serum VEC, serum Vcn, and their combination. Furthermore, we analyzed the relationships between the two proteins and the acute complications of AAD. The findings provide new insight into both the pathogenesis and clinical characteristics of AAD.

\section{Materials And Methods Study population}

We enrolled 100 patients with AAD at the First Affiliated Hospital of China Medical University between March 2017 and July 2019 . Patients with AAD were initially admitted to the Emergency Department for evaluation, diagnosed using CTA within $24 \mathrm{~h}$ of the onset of symptoms, and classified according to the Stanford Standard.(12) The exclusion criteria were chronic aortic dissection, malignancy, autoimmune disorders, severe vascular stenosis, hematological disorders, infectious diseases, coronary heart disorders, severe organ failure, congenital heart disorders, previous aortic surgery, Marfan syndrome, Ehlers-Danlos syndrome, other connective tissue or vascular disorders, and the use of non-steroidal anti-inflammatory drugs or steroids. The demographic and clinical characteristics, risk factors, and laboratory test results for each participant were obtained from their electronic medical records. Laboratory testing was performed according to the hospital laboratory uniform measurement standards, as described previously.

Volunteers who were healthy after physical examination were enrolled in the control group if a diagnosis of AAD was excluded using imaging examination, including CT or magnetic resonance imaging, or both, at admission. Blood samples were obtained from 60 healthy volunteers. The exclusion criteria for the control group were malignancy, infection, a history of the use of medication, and immune-related disorders. Ultimately, 60 control participants were included. The demographic and clinical characteristics of these participants were also collected.

All the participants included in the study gave their written informed consent and the study was approved by the Ethics Committee of the First Hospital of China Medical University (Shenyang, China).

\section{Sample collection and the determination of serum VEC and Ven concentrations}

The participants underwent venipuncture on an empty stomach in the first morning within 24 hours after admission, and the blood samples were collected into EDTA-coated plastic tubes (BD Vacutainer, $5.0 \mathrm{~mL}$ ). The samples were centrifuged to collect plasma (3,000 r/min), which was stored at $-80^{\circ} \mathrm{C}$ for up to 1 year. The serum concentrations of VEC and Vcn were measured using ELISA kits (VEC, R\&D systems, USA; Vcn, Cusabio, Wuhan, China), according to the manufacturers' protocols.

\section{Statistical analysis}

Statistical analyses were performed using SPSS 22.0 (IBM Inc., Armonk, NY, USA). Categorical variables are shown as numbers with percentages, and biochemical and clinical data for the two groups were compared using the chi-square test. Continuous variables are shown as mean values with standard deviations. The relationships between continuous variables were analyzed using Spearman's correlation analysis. Multiple logistic regression models were constructed to assess the relationships of the serum VEC and Vcn concentrations with the risk of AAD, after adjustment for potential confounding factors. Receiver operating characteristic (ROC) curves and the associated areas under the curves (AUCs), based on logistic models, were used to determine the most appropriate cut-off values and assess the diagnostic performance of serum VEC, Vcn, and a combination for AAD. $P \leq 0.05$ (two-sided) was considered to represent statistical significance.

\section{Results}

\section{Baseline clinical characteristics of the participants}

The detailed demographic characteristics and clinical features of the participants with AAD and controls are listed in Table 1. No significant differences were observed in age, sex, body mass index (BMI), the prevalence of diabetes mellitus, and the prevalence of smoking between the controls and participants with $\operatorname{AAD}(p=0.260, p=0.633, p=0.287, p=0.906$, and $p=290$, respectively). Compared with the control group, the participants who had been diagnosed with AAD had significantly higher heart rate, white blood cell (WBC) count, and prevalence of hypertension ( $p<0.001, p<0.001$, and $p=0.001$, respectively), and a lower hemoglobin (Hb) concentration. In the AAD group, 44 participants experienced acute complications (44\%), including refractory pain (34 participants), uncontrollable hypertension despite adequate medical

Page $3 / 12$ 
treatment (22 participants), and poor perfusion of the limbs (12 participants) or viscera (26 participants). Refractory pain and uncontrollable hypertension refer to hypertension and pain that cannot be adequately treated by all existing modern pharmacological methods. Occlusion of celiac trunk, superior mesenteric, inferior mesenteric and/or renal arteries results in severe abdominal pain and decreased urine output leading to metabolic shock later on. When the flow in the distal abdominal aorta or iliac arteries is compromised, patients may complain of painful, pulseless or even plegic and cold lower extremities. All paticipants with AAD received appropriate medication after the onset of symptoms to control pain, heart rate and blood pressure to the normal range. In addition, 77 participants subsequently underwent thoracic endovascular aortic repair (TEVAR).

Table 1

Demographic and characteristics of AAD patients and controls included in this study

\begin{tabular}{|c|c|c|c|}
\hline Variables & Control $(n=60)$ & $\operatorname{AAD}(n=100)$ & $\mathrm{p}$ Value \\
\hline Age, years & $53.75 \pm 1.49$ & $56.09 \pm 1.35$ & 0.26 \\
\hline Male, n(\%) & $48(80)$ & $83(83)$ & 0.633 \\
\hline $\mathrm{BMI}, \mathrm{kg} / \mathrm{m}^{2}$ & $22.00 \pm 0.44$ & $22.68 \pm 0.47$ & 0.287 \\
\hline Hypertension, n(\%) & $25(41.67)$ & $69(69)$ & 0.001 \\
\hline Diabetes mellitus, $n(\%)$ & 8(13.33) & $14(14)$ & 0.906 \\
\hline Smoking, $n(\%)$ & 19(31.67) & $40(40)$ & 0.29 \\
\hline Heart rate, bmp & $78.62 \pm 1.17$ & $88.17 \pm 1.29$ & $<0.001$ \\
\hline WBC, $10^{9} / \mathrm{L}$ & $5.85 \pm 0.10$ & $10.05 \pm 0.38$ & $<0.001$ \\
\hline $\mathrm{Hb}, \mathrm{g} / \mathrm{L}$ & $151.73 \pm 17.59$ & $142.13 \pm 1.75$ & 0.001 \\
\hline Vinculin, $\mathrm{pg} / \mathrm{mL}$ & $102.3 \pm 3.09$ & $145.4 \pm 9.62$ & $<0.001$ \\
\hline VE-Cadherin, ng/uL & $3.15 \pm 0.14$ & $4.49 \pm 0.34$ & 0.028 \\
\hline CRP, mg/L & - & $55.23 \pm 5.18$ & - \\
\hline D-Dimer, ug/mL & - & $6.26 \pm 0.70$ & - \\
\hline \multicolumn{4}{|l|}{ Management in hospital } \\
\hline Medical Therapy & - & $100(100)$ & - \\
\hline TEVAR & - & 77(77) & - \\
\hline Acute phase complications & & $44(44)$ & \\
\hline Refractory pain & - & $34(34)$ & - \\
\hline Uncontrollable hypertension & - & $22(22)$ & - \\
\hline Limb malperfusion & - & $12(12)$ & - \\
\hline Visceral malperfusion & - & $26(26)$ & - \\
\hline
\end{tabular}

\section{Serum Ven and VEC concentrations in AAD patients}

As expected, the serum Vcn concentration was significantly higher in the AAD group than in the control group, as shown in Table 1 and Fig. 1A (145.4 $\pm 9.62 v s .102 .3 \pm 3.09, p<0.001)$. In addition, the serum Vcn concentration was significantly higher in participants with a history of hypertension than in those without $(151.2 \pm 12.26 v s$. $132.7 \pm 14.81, p=0.043$; Fig. 1B). However, no significant difference was observed between participants with acute-phase features that persisted for $<6$ hours or $>6$ hours $(152.9 \pm 14.81$ vs. $136.0 \pm 11.13, p=0.542$; Fig. 1C).

The serum VEC concentration was also significantly higher in the AAD group than in the control group $(4.49 \pm 0.34$ vs. $3.15 \pm 0.14, p=0.028$; Table 1 and Fig. 1D). However, it did not significantly differ between participants with a history of hypertension and those without (151.2 \pm 12.26 vs. $132.7 \pm 14.81, p=0.043$; Fig. $1 \mathrm{E})$, or between participants with acute-phase features that persisted for $<6$ hours or $>6$ hours $(152.9$ \pm 14.81 vs. $136.0 \pm 11.13, p=0.542$; Fig. $1 \mathrm{~F})$. 
The ROC analyses of the diagnostic performances of Vcn, VEC, and their combination for AAD are shown in Table 2 and Fig. 2. The AUCs for Ven and VEC alone were 0.655 and 0.604 , with optimal cut-off values of $128.1 \mathrm{pg} / \mathrm{mL}$ and $3.986 \mathrm{ng} / \mu \mathrm{L}$, respectively. These were associated with sensitivities of $35.0 \%$ and $43.0 \%$, and specificities of $90.0 \%$ and $73.3 \%$, respectively. For the combination of Vcn + VEC, the AUC (0.739) was higher than for Ven or VEC alone, and Ven + VEC yielded a sensitivity of $56.0 \%$, which was also higher than for Ven or VEC alone. The specificity of the combination was $76.7 \%$, which was higher than that for Ven alone, but not for VEC alone.

Table 2

Diagnostic performences of serum Vinculin and VE-Cadherin alone and their combination for AAD detection

\begin{tabular}{|llllll|}
\hline Variables & Cut-off value & AUC(95\% Cl) & Sensitivity & Specificity & p value \\
\hline Vinculin, $\mathrm{pg} / \mathrm{ml}$ & 128.1 & $0.655(0.571-0.739)$ & $35 \%$ & $90 \%$ & 0.001 \\
\hline VE-Cadherin, $\mathrm{ng} / \mathrm{ul}$ & 3.975 & $0.599(0.512-0.686)$ & $43 \%$ & $73.30 \%$ & 0.036 \\
\hline Vinculin + VE-Cadherin & - & $0.661(0.577-0.744)$ & $33 \%$ & $93.33 \%$ & $<0.001$ \\
\hline
\end{tabular}

\section{Relationships of serum Vcn and VEC with blood parameters}

We next evaluated the relationship between the serum Vcn and VEC concentrations, and found a significant correlation between them ( $r=$ $0.207, p=0.039$ ). We also found that serum Vcn significantly correlated with neutrophil count, high-density lipoprotein-cholesterol (HDL-C) concentration, and triglyceride (TG) concentration ( $r=0.202,0.235$, and -0.320 , and $p=0.043, p=0.033$, and $p=0.003$, respectively; Table 3 ). Moreover, there was a significant correlation between the serum concentrations of VEC and D-dimer $(r=0.217, p=0.030 ;$ Table 3$)$. 
Table 3

The correlations between Vinculin and VE-Cadherin levels and labotory tests in AAD patients

\begin{tabular}{|c|c|c|c|c|}
\hline \multirow[t]{2}{*}{ Blood parameters } & \multicolumn{2}{|c|}{ Vinculin (pg/mL) } & \multicolumn{2}{|c|}{ VE-Cadherin (ng/ $\mu \mathrm{L})$} \\
\hline & $\mathrm{R}$ & $\mathrm{p}$ value & $\mathrm{R}$ & $\mathrm{p}$ value \\
\hline \multicolumn{5}{|l|}{ Blood routine } \\
\hline WBC, $\times 10^{9} / \mathrm{L}$ & 0.194 & 0.054 & 0.084 & 0.409 \\
\hline $\mathrm{RBC}, \times 10^{12} / \mathrm{L}$ & -0.047 & 0.649 & -0.023 & 0.826 \\
\hline $\mathrm{HGB}, \mathrm{g} / \mathrm{L}$ & -0.056 & 0.58 & -0.075 & 0.459 \\
\hline $\mathrm{BA}, \times 10^{9} / \mathrm{L}$ & -0.005 & 0.964 & -0.047 & 0.646 \\
\hline $\mathrm{EO}, \times 10^{9} / \mathrm{L}$ & -0.093 & 0.359 & -0.046 & 0.652 \\
\hline $\mathrm{LY}, \times 10^{9} / \mathrm{L}$ & -0.154 & 0.125 & -0.136 & 0.179 \\
\hline $\mathrm{MO}, \times 10^{9} / \mathrm{L}$ & 0.090 & 0.372 & -0.059 & 0.560 \\
\hline $\mathrm{NE}, \times 10^{9} / \mathrm{L}$ & 0.202 & $0.043^{*}$ & 0.111 & 0.270 \\
\hline $\mathrm{MCH}, \mathrm{pg}$ & 0.058 & 0.578 & -0.048 & 0.644 \\
\hline $\mathrm{MCHC}, \mathrm{g} / \mathrm{L}$ & -0.156 & 0.13 & -0.008 & 0.942 \\
\hline MCV, fL & 0.121 & 0.242 & -0.055 & 0.597 \\
\hline $\mathrm{HCT}, \mathrm{L} / \mathrm{L}$ & -0.018 & 0.861 & -0.072 & 0.489 \\
\hline \multicolumn{5}{|c|}{ Blood coagulation function } \\
\hline APTT, s & -0.176 & 0.09 & -0.046 & 0.633 \\
\hline PT, s & -0.041 & 0.693 & -0.092 & 0.376 \\
\hline PTA, \% & -0.019 & 0.855 & 0.086 & 0.405 \\
\hline INR & 0.018 & 0.864 & -0.065 & 0.53 \\
\hline D-Dimer, ug/ml & 0.048 & 0.636 & 0.217 & $0.030 *$ \\
\hline \multicolumn{5}{|c|}{ Cardiovascular injury-related parameters } \\
\hline CK, U/L & -0.007 & 0.952 & 0.17 & 0.162 \\
\hline \multicolumn{5}{|c|}{ Inflammatory response } \\
\hline CRP, mg/L & 0.013 & 0.902 & 0.065 & 0.524 \\
\hline \multicolumn{5}{|l|}{ Liver function } \\
\hline$A L B, g / L$ & -0.023 & 0.828 & 0.034 & 0.741 \\
\hline$A L P, U / L$ & -0.008 & 0.944 & -0.106 & 0.319 \\
\hline $\mathrm{ALT}, \mathrm{U} / \mathrm{L}$ & -0.014 & 0.895 & -0.103 & 0.321 \\
\hline AST, U/L & 0.144 & 0.19 & -0.12 & 0.277 \\
\hline $\mathrm{PA}, \mathrm{mg} / \mathrm{dL}$ & -0.184 & 0.128 & 0.178 & 0.140 \\
\hline $\mathrm{LDH}, \mathrm{U} / \mathrm{L}$ & 0.055 & 0.646 & -0.139 & 0.242 \\
\hline \multicolumn{5}{|l|}{ Serum lipid profile } \\
\hline HDL-C, mmol/L & 0.235 & $0.033 *$ & 0.033 & 0.766 \\
\hline TC, mmol/L & -0.083 & 0.468 & 0.134 & 0.244 \\
\hline $\mathrm{TG}, \mathrm{mmol} / \mathrm{L}$ & -0.32 & $0.003^{* *}$ & 0.069 & 0.535 \\
\hline
\end{tabular}




\begin{tabular}{|c|c|c|c|}
\hline \multirow{2}{*}{$\begin{array}{l}\text { Blood parameters } \\
\text { LDL-C, mmol/L }\end{array}$} & Vinculin (pg/mL) & \multicolumn{2}{|c|}{ VE-Cadherin (ng/ $\mu \mathrm{L})$} \\
\hline & -0.137 & 0.108 & 0.336 \\
\hline \multicolumn{4}{|c|}{$\begin{array}{l}\text { Note, WBC, white blood cells; RBC, red blood cells; HGB, hemoglobin; ; BA, basophil; EO, eosinophil; LY, lymphocyte; MO, monocyte; NE, } \\
\text { neutrophil; MCH, mean corpuscular hemoglobin; MCHC, mean corpuscular hemogobin concentration; MCV, mean corpuscular Volume; } \\
\text { HCT, hematocrit; NLR, neutrophil to lymphocyte ratio; MLR, monocyte to lymphocyte ratio; APT, activated partial thromboplastin time; } \\
\text { PT, prothrombin time; PTA, prothrombin activity, INR, international normalized ratio; CK, creatine kinase; CRP, C-reactive protein; ALB, } \\
\text { albumin; ALP, alkaline phosphatase; ALT, alanine aminotransferase; AST, aspartate aminotransferase; PA, pre-albumin; LDH, lactate } \\
\text { dehydrogenase; HDL-C, high-density lipoprotein-cholesterol; TC, total cholesterol; TG, triglycerides; LDL-C, low-density lipoprotein- } \\
\text { cholesterol. }\end{array}$} \\
\hline$\star P<0.05 ; * \star P<0.01 ; * \star \star P<0.001$. & & & \\
\hline
\end{tabular}

\section{The relationships of serum Ven and VEC with acute preoperative complications of AAD}

We next performed multiple logistic regression analysis to evaluate the relationships of serum Vcn and VEC with the acute preoperative complications of AAD, with adjustment for age, sex, BMI, smoking, hypertension, and diabetes mellitus (Table 4). A high serum Vcn concentration was associated with a higher risk of poor visceral perfusion (diagnosis by computed tomographic angiography) in participants with AAD (odds ratio $[\mathrm{OR}]=1.007$ per unit increase, $95 \%$ confidence interval $[\mathrm{Cl}]=1.001-1.013, p=0.014$ ). In addition, in participants with refractory pain, the adjusted OR for serum VEC concentration increased to 1.172 (95\% Cl: $1.010-1.361, p=0.036$ ), compared with participants without refractory pain.

Table 4

The adjusted logistic regression analysis of Vinculin and VE-Cadherin at baseline and association with AD acute-phase complications

\begin{tabular}{|c|c|c|c|c|c|c|c|c|c|c|}
\hline \multirow[t]{2}{*}{ Vinculin } & \multicolumn{2}{|l|}{ Model 1} & \multicolumn{2}{|l|}{ Model 2} & \multicolumn{6}{|c|}{ Model 3} \\
\hline & $\beta$ & $\begin{array}{l}\text { OR } \\
(95 \% \mathrm{Cl})\end{array}$ & $\mathrm{p}$ Value & & $\beta$ & OR $(95 \% \mathrm{Cl})$ & $\mathrm{p}_{\text {Value }}$ & $\beta$ & OR $(95 \% \mathrm{Cl})$ & $\mathrm{p}_{\text {Value }}$ \\
\hline $\begin{array}{l}\text { Refractory } \\
\text { pain }\end{array}$ & 0.003 & $1.003(0.99$ & $99: 1.007)$ & 0.175 & 0.003 & $1.003(0.998: 1.007)$ & 0.247 & 0.003 & $1.003(0.998: 1.007)$ & 0.262 \\
\hline $\begin{array}{l}\text { Uncontrollable } \\
\text { hypertension }\end{array}$ & 0.005 & $1.005(1.00$ & 01:1.010) & 0.024 & 0.004 & 1.004(0.999:1.009) & 0.086 & 0.004 & 1.004(0.999:1.010) & 0.150 \\
\hline $\begin{array}{l}\text { Limb } \\
\text { malperfusion }\end{array}$ & 0.004 & $1.004(1.00$ & 00:1.009) & 0.075 & 0.003 & $1.003(0.999: 1.008)$ & 0.170 & 0.004 & 1.004(0.999:1.010) & 0.109 \\
\hline $\begin{array}{l}\text { Visceral } \\
\text { malperfusion }\end{array}$ & 0.007 & $1.008(1.00$ & 02:1.013) & 0.006 & 0.007 & $1.007(1.001: 1.013)$ & 0.013 & 0.007 & $1.007(1.001: 1.013)$ & 0.014 \\
\hline \multirow[t]{2}{*}{ VE-Cadherin } & \multicolumn{2}{|l|}{ Model 1} & \multicolumn{2}{|l|}{ Model 2} & & & & \multicolumn{2}{|c|}{ Model 3} & \\
\hline & $\beta$ & OR $(95 \% \mathrm{Cl})$ & & $\mathrm{p}_{\text {Value }}$ & $\beta$ & OR $(95 \% \mathrm{Cl})$ & $\stackrel{p}{\text { Value }}$ & $\beta$ & OR (95\%Cl) & $\begin{array}{l}\mathrm{p} \\
\text { Value }\end{array}$ \\
\hline $\begin{array}{l}\text { Refractory } \\
\text { pain }\end{array}$ & 0.16 & $1.173(1.02$ & 24:1.344) & 0.021 & 0.158 & $1.172(1.011: 1.358)$ & 0.036 & 0.159 & 1.172(1.010:1.361) & 0.036 \\
\hline $\begin{array}{l}\text { Uncontrollable } \\
\text { hypertension }\end{array}$ & 0.069 & $1.072(0.94$ & $45: 1.215)$ & 0.283 & 0.028 & $1.029(0.899: 1.177)$ & 0.680 & 0.027 & 1.028(0.892:1.184) & 0.706 \\
\hline $\begin{array}{l}\text { Limb } \\
\text { malperfusion }\end{array}$ & 0.075 & $1.077(0.92$ & 29:1.249) & 0.323 & 0.039 & $1.040(0.888: 1.218)$ & 0.627 & 0.044 & $1.045(0.889: 1.227)$ & 0.595 \\
\hline $\begin{array}{l}\text { Visceral } \\
\text { malperfusion }\end{array}$ & 0.057 & $1.059(0.93$ & 36:1.197) & 0.364 & 0.026 & $1.027(0.902: 1.168)$ & 0.690 & 0.037 & 1.037(0.905:1.189) & 0.599 \\
\hline
\end{tabular}

\section{Discussion}

To the best of our knowledge, the present study is the first to report that serum Vcn and VEC may represent useful circulating biomarkers of AAD. AAD is a serious disease that requires vascular surgery and is characterized by a tear in the descending aorta, high mortality, and disability. (13) Endothelial injury is considered to be an important component of the pathogenesis of AAD. $(14,15)$ Vcn and VEC play crucial 
roles in the formation and stabilization of epithelial cell-cell adhesion.(16) Therefore, in the present study, the high serum concentrations of Vcn and VEC may reflect the severity of the aortic injury in AAD.

Vcn is an important component of the focal adhesion complex(9) and exists in active and inactive forms. The active form of Vcn is localized to focal adhesions at membranes and participates in their regulation.(17) The recruitment of $V c n$ is important for the maintenance of the epithelial barrier, which is achieved by protecting endothelial junctions from opening during force-dependent remodeling.(11) Zemljic-Harpf and colleagues reported that Vcn deficiency contributes to cardiomyopathy.(18) VEC is an endothelial-specific member of the cadherin family that can maintain the stability of endothelial cell-cell junctions.(19) Recent studies have shown that when endothelial junctions are disturbed, some VEC molecules can be released into the blood in a soluble form. $(20,21)$ The loss of VEC induces pathophysiological conditions, including inflammation, vascular leakage, and tumor-associated angiogenesis. $(22,23)$ It has also been previously reported that Vcn can protect VEC junctions from opening during their force-dependent remodeling.(16)

It has been reported that mechanical stretch can aggravate AAD in a $\beta$-aminopropionitrile-induced rat model.(24) Therefore, increases in the serum concentrations of VEC and Vcn may reflect dysfunction of endothelial junctions. Furthermore, if there are fewer or weaker Vcndependent VEC-based junctions, an appropriate response cannot be mounted to the higher force being exerted on the aortic wall in patients with $A A D$, which may promote its progression.

Previous studies have shown that when the connections between vascular endothelial cells are damaged, the permeability of endothelium increases, and immune cells are able to penetrate the vascular wall, which reduces its integrity and promotes aortic dissection.(25-27) During this process, vascular endothelial inflammation develops, which is characterized by an accumulation of innate immune cells.(28)

Serum C-reactive protein (CRP) and D-dimer concentrations are routinely measured to aid in the diagnosis of AAD. However, the serum CRP concentration is rarely high in the acute phase of onset of $A D$, which implies that it has low diagnostic value for the early diagnosis of $A A D$. $(29,30)$ In addition, the sensitivity and negative predictive value of serum D-dimer concentration are very high at the time of patient admission, but the associated specificity and positive predictive value are much lower.(31-33) Because AAD affects the aortic wall, biomarkers related to injury of the vascular endothelium may be of clinical value.

In the present study, having identified high serum VEC and Vcn concentrations in most of the participants with AAD, we next determined the value of serum VEC and Vcn and their combination for the diagnosis of AAD. According to the ROC curves, both Vcn and VEC have relatively high specificity for the diagnosis of AD, and the specificity of Vcn was higher than that of VEC. However, the sensitivities for the use of both proteins were unsatisfactory ( $35 \%$ and $43 \%$ respectively). Interestingly, the VEC-Vcn combination improved the diagnostic accuracy and sensitivity, and yielded a significantly higher AUC (0.739) than VEC or Vcn alone. These findings suggest that serum VEC and Vcn represent non-invasive markers of $A A D$, and the use of the two in combination may represent a promising means of improving the diagnosis of $A A D$, and especially the differential diagnosis of a high serum D-dimer concentration. We believe that the development of joint diagnostic kits in the future can further improve diagnostic efficiency.

In the present study, we also found that participants with a history of hypertension had a significantly higher serum Ven concentration than those without hypertension. The serum VEC and Vcn concentrations significantly correlated and each concentration correlated with several other blood parameters. VEC concentration significantly correlated with that of D-dimer, and Vcn concentration significantly correlated with neutrophil count, and the serum HDL-C and TG concentrations. D-dimer is a cross-linked fibrin degradation product that appears in the serum after thrombolysis, and neutrophil count may reflect the acute-phase inflammatory status in AAD.(34) In addition, high serum HDL-C and TG concentrations are considered to be the risk factors for cardiovascular disease.(35-37)

Further multiple logistic regression analysis showed that the serum VEC and Vcn concentrations were associated with the prevalence of acute preoperative complications in patients with AAD. Of note, compared with patients with refractory pain, the adjusted OR for VEC concentration in patients without refractory pain increased to 1.172. However, high $\mathrm{Vcn}$ concentration is also indicative of a higher risk of poor visceral perfusion in patients with AD. Thus, although these parameters do not reflect all the aspects of the condition, it is clear that they provide at least a partial indication of the progress of the disease.

The present study had some limitations. First, the changes in the serum VEC and Vcn concentrations were not assessed. Second, the sample size was relatively small. Third, we were not able to measure many blood parameters in the control group. However, the future assessment of tissue expression levels and mechanistic research will provide more detailed information regarding the links between serum VEC and Vcn concentration and AAD.

In conclusion, we have shown that the serum concentrations of both serum Vcn and VEC are significantly higher in patients with AAD. In addition, there were close associations between Vcn and VEC and preoperative complications. Furthermore, we have provided evidence that

Page 8/12 
both VEC or Vcn represent highly specific biomarkers for AAD and may be applicable to the differential diagnosis of AAD.

\section{Declarations}

\section{Conflict of interest}

None.

\section{Funding}

Funding: This work was supported by National Natural Science Foundation of China (grant number: 81970402)

\section{Contributions:}

SYW and YCH: Experimental work.

SYW and YCH Result analysis and manuscript writing.

SYW, YCH, XL, and HJ: Clinical data collection.

JZ: Study design and manuscript revise.

JZ and SJX: Study coordination.

All authors read and approved the final manuscript.

SYW, YCH and JZ: Manuscript revision/review and final approval.

\section{Availability of data and materials}

The data used in this study are available from the corresponding author if

needed.

\section{Ethics approval and consent to participate}

Participants gave written informed consent before the study, and the study

protocol was approved by the Ethics Committee of China Medical University

(CMU), in accordance with the Declaration of Helsinki.

\section{Consent for publication}

Not applicable.

\section{Acknowledgements}

We thank Mark Cleasby, PhD, from Liwen Bianji, Edanz Group China (www.liwenbianji.cn/ac), for editing the English text of a draft of this manuscript.

\section{References}

1. Erbel R, Aboyans V, Boileau C, Bossone E, Bartolomeo RD, Eggebrecht H, et al. 2014 ESC Guidelines on the diagnosis and treatment of aortic diseases: Document covering acute and chronic aortic diseases of the thoracic and abdominal aorta of the adult. The Task Force for the Diagnosis and Treatment of Aortic Diseases of the European Society of Cardiology (ESC). Eur Heart J. 2014;35(41):2873-926.

2. Erbel R, Alfonso F, Boileau C, Dirsch O, Eber B, Haverich A, et al. Diagnosis and management of aortic dissection. Eur Heart J. 2001;22(18):1642-81.

3. Wen D, Zhou XL, Li JJ, Hui RT. Biomarkers in aortic dissection. Clin Chim Acta. 2011;412(9-10):688-95.

4. Giannotta M, Trani M, Dejana E. VE-cadherin and endothelial adherens junctions: active guardians of vascular integrity. Dev Cell. 2013;26(5):441-54. 
5. Harris ES, Nelson WJ. VE-cadherin: at the front, center, and sides of endothelial cell organization and function. Curr Opin Cell Biol. 2010;22(5):651-8.

6. Lee HT, Sharek L, O'Brien ET, Urbina FL, Gupton SL, Superfine R, et al. Vinculin and metavinculin exhibit distinct effects on focal adhesion properties, cell migration, and mechanotransduction. PLoS One. 2019;14(9):e0221962.

7. Alahari MMaSK. Cell matrix adhesions in cancer: The proteins that form the glue. Oncotarget. 2017;8(29):48471-87.

8. Zhang M, Liu P, Xu F, He Y, Xie X, Jiang X. Vinculin promotes gastric cancer proliferation and migration and predicts poor prognosis in patients with gastric cancer. J Cell Biochem. 2019;120(8):14107-15.

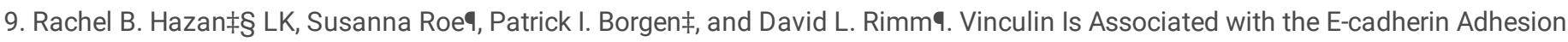
Complex. THE JOURNAL OF BIOLOGICAL CHEMISTRY. 1997;272(December 19):32448-53.

10. Goldmann WH, Ingber DE. Intact vinculin protein is required for control of cell shape, cell mechanics, and rac-dependent lamellipodia formation. Biochem Biophys Res Commun. 2002;290(2):749-55.

11. Twiss F, Le Duc Q, Van Der Horst S, Tabdili H, Van Der Krogt G, Wang N, et al. Vinculin-dependent Cadherin mechanosensing regulates efficient epithelial barrier formation. Biol Open. 2012;1(11):1128-40.

12. Nienaber CA, Eagle KA. Aortic dissection: new frontiers in diagnosis and management: Part I: from etiology to diagnostic strategies. Circulation. 2003;108(5):628-35.

13. Nienaber CA, Clough RE, Sakalihasan N, Suzuki T, Gibbs R, Mussa F, et al. Aortic dissection. Nat Rev Dis Primers. $2016 ; 2: 16053$.

14. He R, Guo DC, Estrera AL, Safi HJ, Huynh TT, Yin Z, et al. Characterization of the inflammatory and apoptotic cells in the aortas of patients with ascending thoracic aortic aneurysms and dissections. J Thorac Cardiovasc Surg. 2006;131(3):671-8.

15. Luo F, Zhou XL, Li JJ, Hui RT. Inflammatory response is associated with aortic dissection. Ageing Res Rev. 2009;8(1):31-5.

16. Huveneers S, Oldenburg J, Spanjaard E, van der Krogt G, Grigoriev I, Akhmanova A, et al. Vinculin associates with endothelial VEcadherin junctions to control force-dependent remodeling. J Cell Biol. 2012;196(5):641-52.

17. Beppu M, Sawai S, Satoh M, Mori M, Kazami T, Misawa S, et al. Autoantibodies against vinculin in patients with chronic inflammatory demyelinating polyneuropathy. J Neuroimmunol. 2015;287:9-15.

18. Zemljic-Harpf AE, Miller JC, Henderson SA, Wright AT, Manso AM, Elsherif L, et al. Cardiac-myocyte-specific excision of the vinculin gene disrupts cellular junctions, causing sudden death or dilated cardiomyopathy. Mol Cell Biol. 2007;27(21):7522-37.

19. Dejana E, Giampietro C. Vascular endothelial-cadherin and vascular stability. Curr Opin Hematol. 2012;19(3):218-23.

20. Sidibe A, Mannic T, Arboleas M, Subileau M, Gulino-Debrac D, Bouillet L, et al. Soluble VE-cadherin in rheumatoid arthritis patients correlates with disease activity: evidence for tumor necrosis factor alpha-induced VE-cadherin cleavage. Arthritis Rheum. 2012;64(1):7787.

21. Vilgrain I, Sidibe A, Polena H, Cand F, Mannic T, Arboleas M, et al. Evidence for post-translational processing of vascular endothelial (VE)cadherin in brain tumors: towards a candidate biomarker. PLoS One. 2013;8(12):e80056.

22. Baluk P, Hashizume H, McDonald DM. Cellular abnormalities of blood vessels as targets in cancer. Curr Opin Genet Dev. 2005;15(1):10211.

23. Weis SM. Vascular Permeability in Cardiovascular Disease and Cancer. Current Opinion in Hematology. 2008;15:7.

24. Hu Y, Lu L, Qiu Z, Huang Q, Chen Y, Chen L. Mechanical stretch aggravates aortic dissection by regulating MAPK pathway and the expression of MMP-9 and inflammation factors. Biomed Pharmacother. 2018;108:1294-302.

25. Keck T, Balcom JHt, Fernandez-del Castillo C, Antoniu BA, Warshaw AL. Matrix metalloproteinase-9 promotes neutrophil migration and alveolar capillary leakage in pancreatitis-associated lung injury in the rat. Gastroenterology. 2002;122(1):188-201.

26. del Porto F, Proietta M, Tritapepe L, Miraldi F, Koverech A, Cardelli P, et al. Inflammation and immune response in acute aortic dissection. Ann Med. 2010;42(8):622-9.

27. Ngok SP, Geyer R, Liu M, Kourtidis A, Agrawal S, Wu C, et al. VEGF and Angiopoietin-1 exert opposing effects on cell junctions by regulating the Rho GEF Syx. J Cell Biol. 2012;199(7):1103-15.

28. Edens HA PC. Neutrophil transendothelial migration and alteration in vascular permeabilityFocus on neutrophil-derived azurocidin. Curr Opin Hematol. 2003;10(1):25-30.

29. Wen D, Du X, Dong JZ, Zhou XL, Ma CS. Value of D-dimer and C reactive protein in predicting inhospital death in acute aortic dissection. Heart. 2013;99(16):1192-7.

30. Mori K, Tamune H, Tanaka H, Nakamura M. Admission Values of D-dimer and C-reactive Protein (CRP) Predict the Long-term Outcomes in Acute Aortic Dissection. Intern Med. 2016;55(14):1837-43. 
31. OhImann P, Faure A, Morel O, Petit H, Kabbaj H, Meyer N, et al. Diagnostic and prognostic value of circulating D-Dimers in patients with acute aortic dissection. Crit Care Med. 2006;34(5):1358-64.

32. Shimony A, Filion KB, Mottillo S, Dourian T, Eisenberg MJ. Meta-analysis of usefulness of d-dimer to diagnose acute aortic dissection. Am J Cardiol. 2011;107(8):1227-34.

33. Fulvio Morello PP, Miroslav Novak \& Peter Kruzliak. Biomarkers for diagnosis and prognostic stratification of aortic dissection challenges and perspectives. Biomarkers Med. 2014;8(7):931-41.

34. Zhang J, Jiang Y, Gao C, Feng J, Wang A. Risk factors for hospital death in patients with acute aortic dissection. Heart Lung Circ. 2015;24(4):348-53.

35. Turak O, Afsar B, Ozcan F, Oksuz F, Mendi MA, Yayla C, et al. The Role of Plasma Triglyceride/High-Density Lipoprotein Cholesterol Ratio to Predict New Cardiovascular Events in Essential Hypertensive Patients. J Clin Hypertens (Greenwich). 2016;18(8):772-7.

36. Girona J, Amigo N, Ibarretxe D, Plana N, Rodriguez-Borjabad C, Heras M, et al. HDL Triglycerides: A New Marker of Metabolic and Cardiovascular Risk. Int J Mol Sci. 2019;20(13).

37. Zhou Y, Yang G, He H, Pan X, Peng W, Chai X. Triglyceride/High-Density Lipoprotein Cholesterol Ratio Is Associated with In-Hospital Mortality in Acute Type B Aortic Dissection. Biomed Res Int. 2020;2020:5419846.

\section{Figures}

A

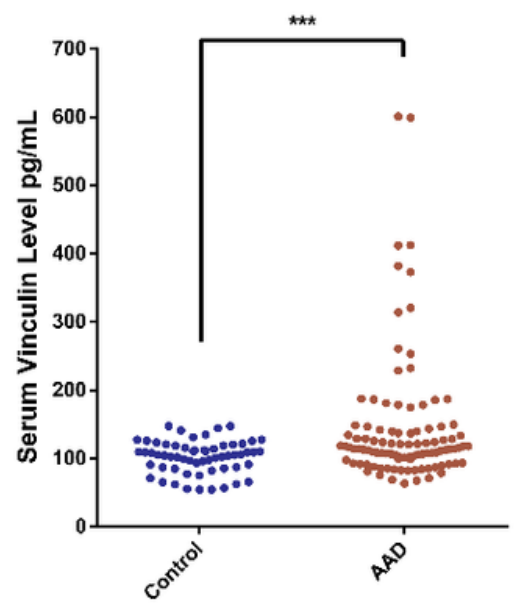

D

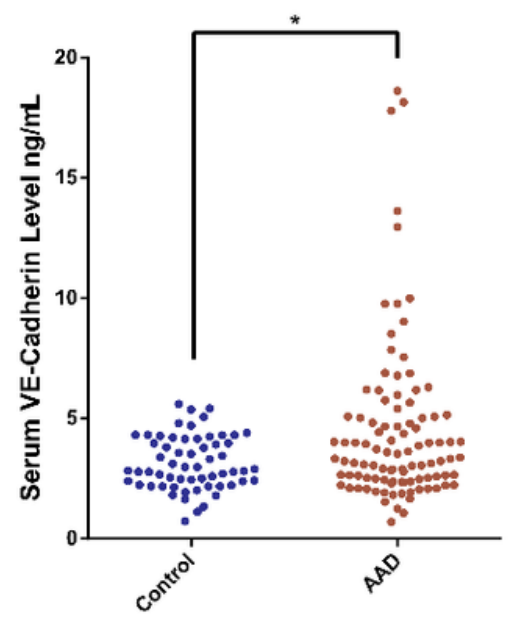

B

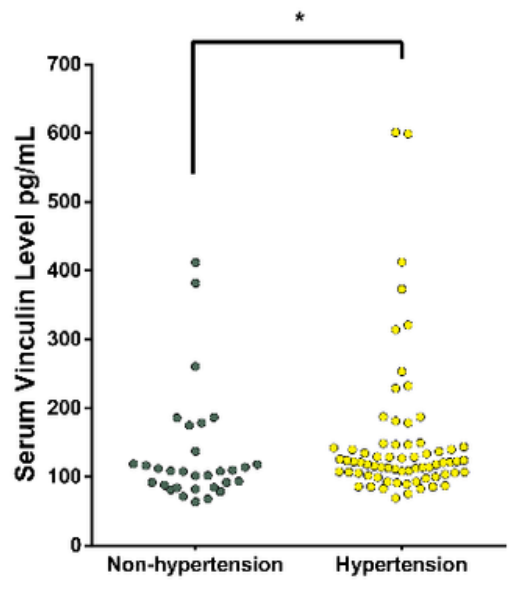

E

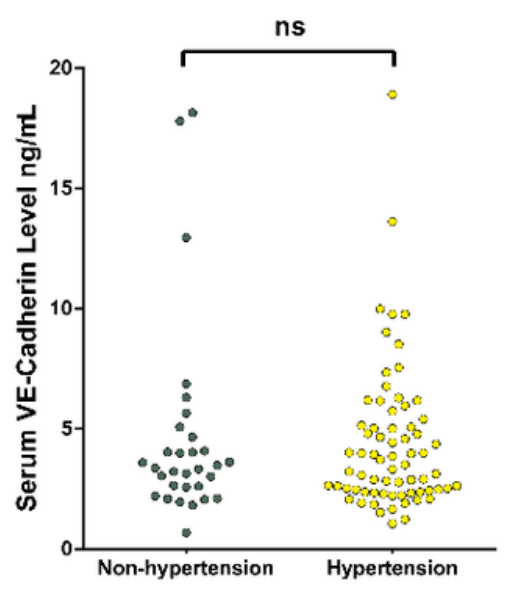

C
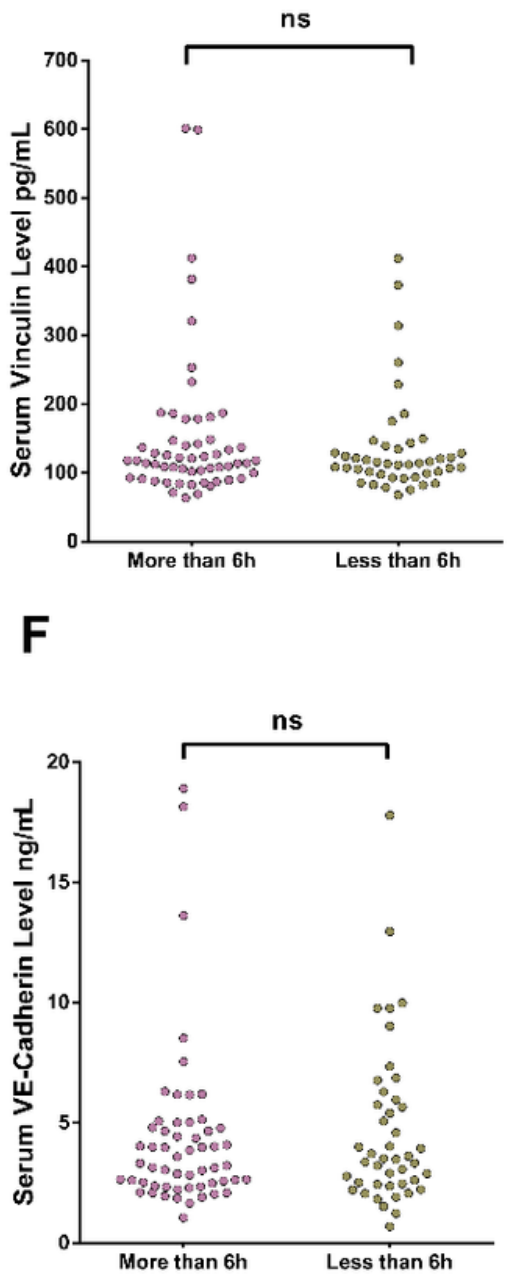

Figure 1 
Analysis of serum Vcn and VEC concentrations in different groups. The horizontal axis represents serum Vcn concentrations in mg/L and the vertical axis represents (A) control, AAD (B) Non-Hypertension, Hypertension, (C) more than 6h, less than 6h. The horizontal axis

represents serum VEC concentrations in $\mathrm{mg} / \mathrm{L}$ and the vertical axis represents (D) control, $A A D$, (E) Non-Hypertension, Hypertension, and (F) more than $6 \mathrm{~h}$, less than $6 \mathrm{~h}$.

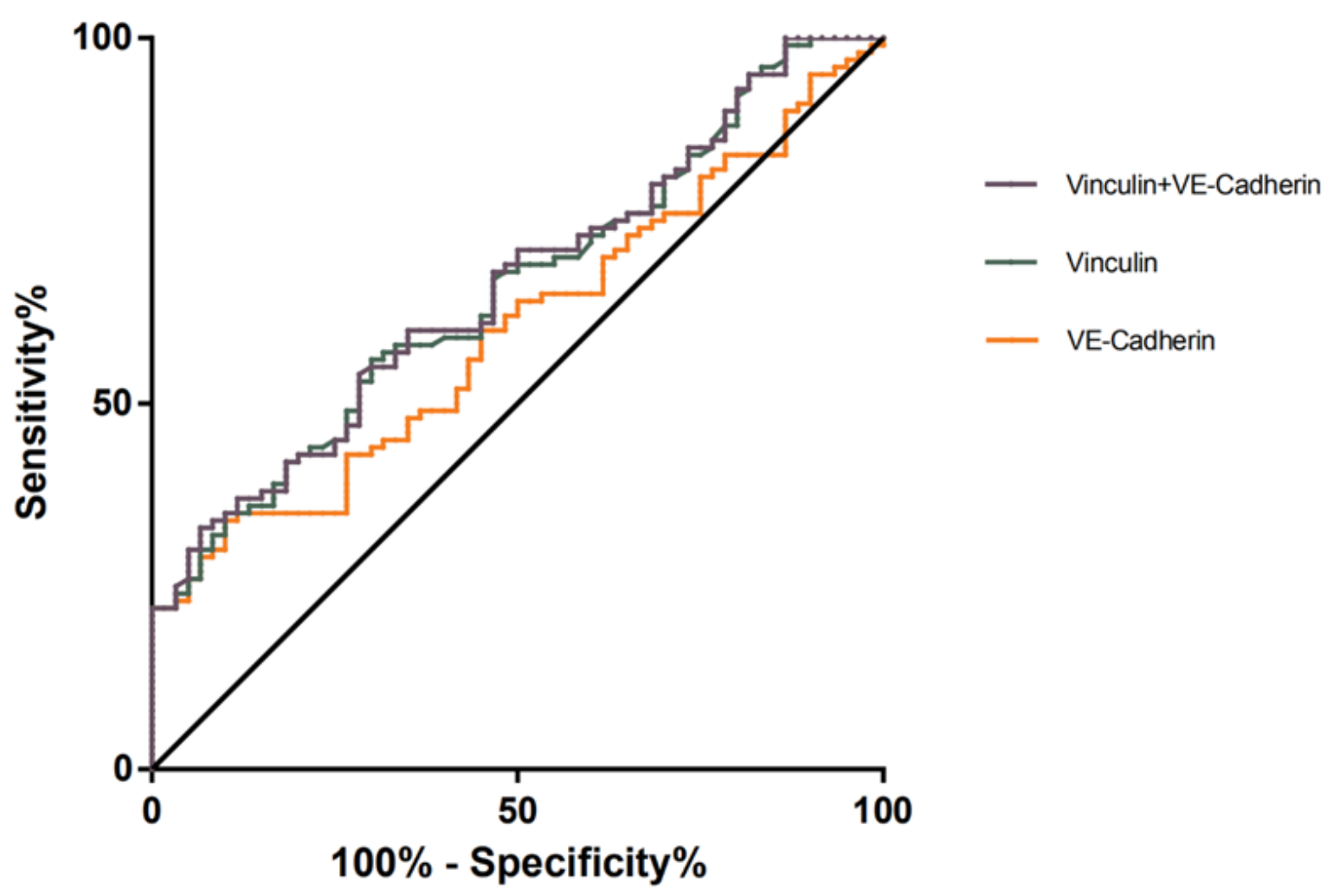

Figure 2

ROC analysis of Vcn, VEC, and their combination for the evaluation of AAD. The vertical axis represents the sensitivity and the horizontal axis represents the 1-specificity. 